\title{
Mindsets and pre-conscious open-mindedness to incidental information ${ }^{\text {th }}$
}

\author{
Kentaro Fujita $^{\mathrm{a}, *}$, Peter M. Gollwitzer ${ }^{\mathrm{a}, \mathrm{b}}$, Gabriele Oettingen ${ }^{\mathrm{a}, \mathrm{c}}$ \\ a Department of Psychology, New York University, New York, USA

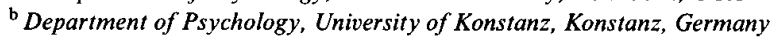 \\ ${ }^{c}$ Department of Psychology, University of Hamburg, Hamburg, Germany
}

\begin{abstract}
Mindset theory (Gollwitzer, 1990) proposes that deliberative mindsets are marked by more open-minded processing of information, whereas implemental mindsets are characterized by more closed-minded processing. Accordingly, deliberative and implemental mindsets should differ in selective processing of incidental information when performing a central task. In three experiments, participants in deliberative and implemental mindsets performed a computer task while randomly presented incidental, unavoidable words. A subsequent recognition memory test assessed selective processing of these incidental words. Results revealed that deliberative mindsets led to superior recognition memory, suggesting increased open-mindedness to processing incidental information. Implications for mindset and goal theories are discussed.
\end{abstract}

\section{Introduction}

The cognitive processes that support and maintain goal pursuit have become a central issue among researchers studying motivation (Gollwitzer \& Bargh, 1994; Kruglanski et al., 2002; Oettingen \& Gollwitzer, 2001; Sorrentino \& Higgins, 1986). Mindset theory was one of the first theories to describe the complex interplay between cognitive and motivational processes, and continues to be one of the most prominent and influential frameworks (Gollwitzer, 1990).

\footnotetext{
* This research was supported by a National Science Foundation Graduate Student Fellowship, and the Interdisciplinary Center for Research on Intention and Intentionality at the University of Konstanz. Thanks go to Suah Lee, Susan Nacorda, and Julia Travis for their assistance in data collection. Special thanks also go to K.C. McCulloch for extensive discussion of ideas. Members of the NYU Motivation Lab provided valuable feedback on an earlier version of this paper.

* Corresponding author

E-mail address: kf348@nyu.edu (K. Fujita)
}

This theoretical model posits that there are multiple stages of goal pursuit, called action phases, which individuals must successfully navigate to attain a goal. In particular, the theory assumes that each phase is characterized by a distinct task that must be accomplished. The two most important of these tasks are choosing a goal and implementing a chosen goal. Whereas individuals must deliberate between the many wishes and desires they might have when choosing a goal, they must plan out when and how to act to implement a chosen goal. Central to the theory is the notion that actively choosing a goal and planning the implementation of a goal activates distinct mindsets, or cognitive procedures, that promote successful navigation through the respective action phases. The studies reported in this paper build upon and advance a body of research that has demonstrated the theoretical and empirical distinction between deliberative and implemental mindsets; that is, differences in information processing when one is engaged in choosing a goal as compared to implementing a chosen goal. 


\section{Empirical support for mindset theory}

A great deal of empirical research has explored the unique cognitive features of deliberative and implemental mindsets (summaries by Gollwitzer \& Bayer, 1999; Gollwitzer, Fujita, \& Oettingen, 2004), those cognitive procedures associated with choosing a goal and those procedures associated with planning and executing actions relevant to a chosen goal, respectively. This research has demonstrated mindset differences primarily in two domains: cognitive tuning and biased inferences.

Thought-sampling research (Heckhausen \& Gollwitzer 1987; Puca \& Schmalt, 2001; Taylor \& Gollwitzer, 1995) has shown that the thoughts of individuals in a deliberative mindset are tuned to expectancy-value considerations, focusing on issues of goal desirability and feasibility. The thoughts of those in implemental mindsets, on the other hand, are dominated by thoughts of the when, where, and how of goal implementation. Individuals in a deliberative mindset also selectively process and attend to expectancyvalue information, whereas those in an implemental mindset attend to goal implementation information (Gollwitzer, Heckhausen, \& Steller, 1990). These results suggest that the deliberative and implemental mindsets are cognitively tuned to distinct concerns, namely choosing between goals and implementing a chosen goal, respectively.

In addition to activating action phase-appropriate thought content, mindset theory suggests that deliberative mindsets should be characterized by an even-handed (impartial) and objective (realistic) analysis of information to allow for good goal decisions (i.e., select goals that are both highly desirable and feasible). In contrast, as questioning one's decision and commitment to a goal can undermine efforts to attain the chosen goal, implemental mindsets should foster biased information processing that reinforces and supports one's intention to reach the goal. Indeed, a great deal of research has shown that the implemental mindset, as compared to the deliberative mindset, leads to more biased positive evaluations and higher expectations of goal success (Armor \& Taylor, 2003; Gagné \& Lydon, 2001; Gollwitzer \& Kinney, 1989; Puca, 2001; Taylor \& Gollwitzer, 1995). Taylor and Gollwitzer (1995, Study 3) have shown, for example, that in contrast to individuals in a deliberative mindset who engage in balanced considerations of pros and cons while making a decision, individuals in an implemental mindset consider pros five times more frequently than cons. Those in implemental mindsets are also more likely to overestimate the amount of control they have over their environment ("illusions of control"; Gollwitzer \& Kinney, 1989) and how well they can perform on various tasks (Puca, 2001), whereas those in deliberative mindsets are more realistic (i.e., accurate). These findings suggest that as one progresses from choosing a goal to actually implementing a chosen goal, individuals make more biased inferences to defend their goal decision and to protect the realization of the chosen goal.

\section{Mindsets and open-mindedness}

Beyond differences in cognitive tuning and biased inferences, deliberative and implemental mindsets should also differ in openness to information. Task analysis of the demands of making a goal decision suggests that deliberative mindsets should be associated with enhanced receptivity to all sources and types of information. To make good decisions, one should be open to any available information that might potentially inform one's decision-making. One should be careful not to dismiss information prematurely as it may ultimately be useful or helpful in making good goal decisions. Implemental mindsets, in contrast, should be associated with more selective information processing. Once a goal is set, successful goal implementation requires more particular filtering of information, selectively processing goal-relevant stimuli while ignoring goal-irrelevant stimuli (e.g., Gollwitzer, 1990; Kuhl, 1984). For these reasons, the deliberative mindset should be associated with greater openness to information incidental to one's goals.

An early study by Heckhausen and Gollwitzer (1987, Study 2) bears some relevance to this hypothesis. Participants were interrupted either while they were deliberating a choice between two different creativity tests (deliberative mindset), or just after having chosen one of them (implemental mindset), and verbally presented with lists of 5-7 one-syllable nouns (e.g., house, art, and tree). Immediately after each list had been presented, participants had to recall the words in order. Participants' performance in this task was used to compute their working memory span (i.e., noun span), and results indicated that deliberative mindset participants evidenced a broader span (about half a word more) than implemental mindset participants.

The superior noun span by deliberative as compared to implemental mindset participants, however, only suggests that deliberative mindset participants are more capable of storing information (i.e., they have a broader working memory span). Although broadened working memory suggests an enhanced capacity to process information, it does not directly address the hypothesis that deliberative mindsets, as compared to implemental mindsets, are associated with heightened processing of information that is incidental to one's goals. The information in the word lists used in the Heckhausen and Gollwitzer (1987) study cannot be considered incidental. Participants were explicitly asked to correctly reproduce as many words as possible of each presented word list. Moreover, broader working memory span by itself does not necessarily lead to more or less selective processing of incidental information. Accordingly, whereas the Heckhausen and Gollwitzer study demonstrated mindset differences in working memory capacity, the present line of research attempts a critical, more specific test of the hypothesis that there are differences between the deliberative and implemental mindset in the selective processing of incidental information. 


\section{The present studies}

The present studies were designed to test the processing of incidental information in deliberative and implemental mindsets. Specifically, we were interested in documenting differences in selective processing of information incidental to performing a central task at hand (see below). An additional aim of this study was to show that these changes in selective processing occur preconsciously (i.e., without conscious intent or regulation). Selective processing of information can be driven by conscious information search and exposure strategies. For example, an individual might intentionally avert their eyes away from stimuli they deem to be task-irrelevant and distracting. We were interested, however, in those cognitive procedures that occur without the explicit intention or knowledge of the individual. That is, are there pre-conscious processes associated with deliberative and implemental mindsets that help guide an individual's selective processing of incidental information?

We used a paradigm in which participants performed a primary task while presented with incidental information as distractions or impairments to performance on the primary task. Such a paradigm makes it clear to both researchers and participants what is central and incidental while performing the task at hand. Moreover, it was necessary to bypass conscious information search and exposure strategies (active avoidance strategies such as averting one's gaze) to find evidence for pre-conscious rather than conscious differences in cognitive procedures between mindsets. Accordingly, to measure differences in selective processing between mindsets, we created a computer performance task that required participants to attend to the center of the screen. While performing the task (which involved counting objects that were presented in the middle of the screen), participants were briefly presented with words incidental to the task at hand prior to half of the trials. Each word was presented randomly in the same location as materials for the primary performance task (i.e., in the center of their visual field) to prevent strategic anticipation and avoidance. Moreover, the stimuli were presented for only $300 \mathrm{~ms}$ before being replaced by the primary performance task materials, a presentation time generally considered to be too fast for interventions by conscious intent (Bargh \& Chartrand, 2000; Greenwald \& Banaji, 1995; Neely, 1977). Participants were then given a surprise recognition memory test of the incidental words. As participants could not avoid exposure to these words via conscious information search and exposure strategies, any differences in the processing of incidental stimuli between conditions can be attributed to pre-conscious procedures of the mindsets. If deliberative and implemental mindsets differ in degree of selective processing, there should be differences in performance on the recognition memory test.

We used this paradigm in all three experiments presented in this paper, utilizing recognition memory task performance as our measure of selective processing. To induce deliberative and implemental mindsets, in Study 1 we had participants complete the performance task described above while in the midst of deliberating a decision or immediately after making such a decision (e.g., Heckhausen \& Gollwitzer, 1987; Gollwitzer \& Kinney, 1989, Study 1). Participants were given the illusion of being able to choose between two versions of the performance task. Half of them chose immediately which version to perform (implemental condition), while the other half was asked to delay their decision until after they had "tried out" both versions (deliberative condition). In reality, regardless of condition or choice of task, both groups performed the same task (the performance and measurement tasks described above). To replicate and extend our findings, in Studies 2 and 3 we used a different mindset manipulation, inducing mindsets with a classic mindset priming technique (e.g., Gollwitzer \& Kinney, 1989, Study 2; Taylor \& Gollwitzer, 1995). Participants were asked to deliberate over a goal decision or plan out the implementation of a goal decision in an ostensibly "separate" study. In addition, Study 3 included a control condition in which participants engaged in a mindset-neutral thought-listing task. Participants in both Studies 2 and 3 were then asked to complete the performance and recognition memory tasks described previously to measure the indirect or "carry-over" effects of each respective mindset. Across all three studies, we expected differences between deliberative and implemental mindsets on recognition memory performance, demonstrating changes in selective processing (and hence pre-conscious open- and closedminded processing of incidental information) as a function of these mindsets.

\section{Study 1}

\section{Method}

\section{Participants}

Forty-six students at New York University participated in the study in exchange for course credit. Participants were randomly assigned to condition, with 24 in the deliberative mindset condition and 22 in the implemental mindset condition.

\section{Materials and procedures}

Participants were run individually or in groups of two by one of two experimenters. ${ }^{1}$ They were each seated in front of a computer inside a soundproof cubicle, which prevented them from seeing and hearing any other participant. They were all told that they were participating in a study that was ostensibly designed to measure their concentration potential. Participants were then informed that there were two different computer tasks designed for this purpose, one based on "verbal stimuli" and the other based on "spatial stimuli." The experimenter carefully explained to all partic-

\footnotetext{
1 Preliminary analyses indicated that there were no significant effects of experimenter or gender on any of the measures in all studies. All subsequent analyses were collapsed across these variables.
} 
ipants that they could demonstrate their true potential only if they picked the task that was "right" for them. Unbeknownst to the participants, the two "different" computer tasks were the same task. Participants were presented with the illusion of two tasks to create an experimental situation in which they had to make a goal decision (for similar manipulations of mindsets, see Heckhausen \& Gollwitzer, 1987; Gollwitzer \& Kinney, 1989, Study 1; Gollwitzer et al., 1990, Study 1).

Mindset manipulations. Participants in the deliberative mindset condition were instructed to suspend any preconceptions about whether they preferred verbal or spatial stimuli. They were told that the best decision could be made only after they had tried each type of task. While trying out the tasks, participants were instructed to consider carefully whether it was the right task for them, but not to make up their minds until they had performed both. They were then given a choice which task they wanted to try first. The task they subsequently performed was the same task performed by the implemental mindset condition. To prevent a premature goal decision made on the basis of the choice of which task to try first, after participants indicated their choice, they were reminded to ask themselves continually whether they felt that the task they were performing would most accurately demonstrate their concentration potential. In this way, participants were instructed to continue deliberating over their goal decision while performing the task. When participants were finished, they were informed that they would not have to perform the other task nor make a final decision as to which task they wanted to perform. They then continued to the next part of the experiment.

Participants in the implemental mindset condition were asked to think about the activities they commonly engage in, and asked whether they would feel more comfortable with verbal or spatial tasks. They were urged to think deeply about this decision and to avoid impulsive choices, as they would not be able to reverse their decision. Once participants had made their decision and told the experimenter, they performed the appropriate task (which again, was the same task regardless of decision). In this way, participants in the implemental mindset condition performed the task for which they had made a goal decision.

Performance task. After participants indicated their preference, the experimenter set up a computerized version of the d2 Mental Concentration Task (Brickenkamp, 1981). In this task, strings of d's and p's were presented in the center of the computer screen. Each letter string of 12 letters in 12point font was presented in black ink against a white background. Above and below each letter were one, two, or no apostrophes. The participant's task was to count as quickly and as accurately as possible the number of d's that had a total of two apostrophes in a given string. Responses were indicated by pressing one of the four buttons on a response box, labeled with the numbers $4-7$. Each button response had an equivalent probability of being correct. Between tri- als, a blank white screen was presented for $1 \mathrm{~s}$. Participants were presented with three blocks of 28 trials for a total of 84 trials, of which the first block were treated as practice trials and excluded from analysis.

Randomly before half of the actual timed trials, participants were presented with incidental words. Words were a mix of abstract and concrete words (e.g., BONE, ALWAYS, FLAG, EVERY). The words were presented in the center of the screen in black ink 12-point font in all capital letters. Each was presented for $300 \mathrm{~ms}$, and then immediately followed by a string of d's and p's (the d2 Mental Concentration Task stimuli). A total of 28 words were presented. The words were carefully chosen so as to prevent any semantic associations between the stimuli and the performance test. No mention of the incidental words was made to the participant prior to the task. Probing questionnaires (see below) and post-experiment interviews confirmed that participants assumed that they were presented as distractions. ${ }^{2}$

When participants completed the computerized concentration task, they were asked to complete a series of penand-paper questionnaires. Participants first completed several items designed to account for potential alternative explanations for the predicted pattern of results. Descriptions of these measures and the rationale for including them are described in more detail in the Results section. After completing all the measures (about $5 \mathrm{~min}$ ), participants were probed for suspicion regarding the experimental manipulations. They were also asked to guess what function they thought the words in the $\mathrm{d} 2$ Mental Concentration Task had.

Recognition memory task. Participants were then presented with a surprise computerized recognition memory test. In the center of the computer screen, 56 words were presented one at a time. Half of these words (28) were the original words presented during the performance task. An additional 28 words matched in word length, concreteness, and familiarity, were presented as foils (Kucera \& Francis, 1967). Participants were asked to indicate as quickly as they could whether or not the word they saw was presented in the previous performance task. Each word remained on the

\footnotetext{
2 In all three studies, participants who acknowledged that they anticipated the recognition memory test for the incidental stimuli were excluded from the study as their data are difficult to interpret. As they believed that attending to the words to be of primary importance to the goal of performing the computer task, it seems problematic to include their data in an analysis of selective information processing of incidental stimuli. Participants who anticipated the recognition memory test were evenly distributed between conditions (two each from the deliberative and implemental conditions in Study 1; three and five from the deliberative and implemental mindset conditions, respectively, for Study $2 ; 10,5$, and 9 from the deliberative, implemental, and control conditions in Study 3 ). The unusually high number of participants who anticipated the recognition memory test in Study 3 is due in large part to changes in the participant pool. In the fall of 2004, students in more advanced psychology courses were included in the pool, many of whom were aware of standard psychology experimental paradigms.
} 
screen until the participant responded. The order of the words was completely randomized. Upon completion of the recognition test, participants were fully debriefed and dismissed.

\section{Results}

\section{d2 mental concentration task performance}

Performance on the $\mathrm{d} 2$ Mental Concentration Task by mindset was measured using two variables: number of errors and response latencies. Response latencies were transformed using a base-10 logarithmic transformation to correct for excessive skew. A 2 (condition: deliberative vs. implemental) $\times 2$ (trial: word vs. no word) mixed design ANOVAs response latencies revealed only the main effect of trial: $F(1,42)=18.16, p<.001, r=.55 .^{3}$ Participants responded more slowly to trials preceded by words $(M=7981 \mathrm{~ms}, \quad S D=1847)$ than those that were not $(M=7384 \mathrm{~ms}, S D=1401))^{4}$ There were no significant differences with respect to number of errors.

\section{Recognition memory test performance}

Performance on the recognition memory test was analyzed in two ways: accuracy and response latencies. To analyze accuracy, the discriminability index, $A^{\prime}$ was calculated for each participant on the basis of their correct hit and false alarm rates in the recognition task (Pollack \& Norman, 1964). The traditionally used discriminability index, $d^{\prime}$, could not be used as false alarm rates were equal to zero on some occasions. The nonparametric $A^{\prime}$ has been shown to be highly correlated to the parametric $d^{\prime}$ (Snodgrass, Volvovitz, \& Walfish, 1972). To analyze response latencies, all latencies less than $300 \mathrm{~ms}$ and greater than $3000 \mathrm{~ms}$ were excluded from the analysis (Goschke \& Kuhl, 1993). This accounted for less than $4 \%$ of the data. Response latencies were then log-transformed to correct for excessive skew. Means of these log-transformed response latencies associated only with correct responses (i.e., hits and correct rejections) were then calculated for each participant and entered into the analysis (Anderson, 1983; Goschke \& Kuhl, 1993).

Recognition accuracy between the deliberative $(M=.77$, $S D=.078)$ and implemental $(M=.75, S D=.060)$ mindset conditions, although in the predicted direction, was not statistically different, $t(44)=1.12, p=.27, r=.17$. As predicted, however, the difference in response latencies between mindset conditions was statistically significant, $t(44)=2.01$, $p=.05, r=.29$. Participants in the deliberative mindset condition $(M=974 \mathrm{~ms}, S D=160)$ required less time to recognize the incidental words presented during the $\mathrm{d} 2$ Mental Concentration Task than did participants in the implemental mindset condition $(M=1162 \mathrm{~ms}, S D=380)$.

\footnotetext{
${ }^{3}$ Discrepancies in the degrees of freedom for the concentration task are due to missing data due to computer error. The data from two subjects are missing from Study 1, and one subject from Study 2.

${ }^{4}$ For ease of interpretation, throughout this paper, we report the original untransformed means and standard deviations for response latencies.
}

\section{Alternate explanations}

We included several measures in our study design to account for possible alternate explanations for the obtained pattern of results. These re-interpretations of our data would suggest that the differences in response latencies were not a function of mindsets, but rather differences in degree of motivation, self-efficacy, or type of motivation. We review each of these explanations in turn.

Degree of motivation. One possible alternate interpretation would suggest that the differences in recognition test performance resulted not from differences in mindset, but rather in the magnitude of motivation the participants experienced in the two conditions. Recall that while participants in the implemental mindset chose which task they wanted to perform, participants in the deliberative mindset merely chose which task they wanted to try first. One might argue that participants in the latter condition did not take the task as seriously as the former. From this perspective, differences between conditions on the recognition memory test would have resulted from one group being more motivated than the other. If this were true, one might not be surprised to see that the deliberative mindset participants processed incidental words to a greater extent than those in the implemental mindset if they were less motivated to perform well on the central task.

To address this alternate account, we compared responses to the two items tapping into participants' commitment to performing well on the computer task ("How committed were you to performing well on the task?" and "How upset would you be if you performed poorly on the task?"). Participants responded using 9-item Likert-type scales, with $l=$ not at all and $9=$ very much so. There were no significant differences in how committed participants were to success, $t(44)=1.05, p=.30, r=.16$, nor how upset they would be with failure, $t(44)=.74, p=.46, r=.11$. In addition, neither variable was significantly correlated with response latency on the recognition memory task $(r=-.03$, $p=.83 ; r=-.18, p=.23$, respectively). These results indicate that the data do not support the contention that the two conditions differed in the magnitude of their commitment or motivation to the concentration task, and therefore suggest that an alternative account citing differences in these variables is not tenable.

Self-efficacy. Another alternate explanation of the results would suggest that differences in the instructions during the mindset manipulation might have altered participants' sense of self-efficacy. By having participants in the deliberative mindset deliberate over which task they wanted to perform, this account would propose that the manipulation might have inadvertently suggested that they were less capable than those participants in the implemental mindset condition. When an individual feels less capable of performing a task, there might be reason to believe that they attend less carefully to the task and are more likely to process incidental information. 
To address this alternate account, we compared responses to an item measuring self-efficacy ("To what extent did you feel you had the skills and abilities necessary to perform well on this task?"). Participants responded using 9-item Likert-type scales, with $l=$ not at all and $9=$ very much so. Responses to this item did not differ significantly between condition, $t(44)=.74, p=.46, r=.11$. Efficacy was also not significantly correlated with response latency on the recognition test $(r=-.03, p=.87)$. These analyses indicate that the data do not support an alternate account of the results that postulate differences in selfefficacy.

Type of motivation. A third alternate explanation of the results would suggest that participants experienced differing types of motivation while performing the computer task. This account would propose that the instructions given to participants in the deliberative mindset condition led them to be motivated for different reasons than those in the implemental mindset condition. Whereas participants in the implemental mindset condition may have felt more internally motivated when they were given the choice of tasks to perform, participants in the deliberative mindset condition may have felt more externally motivated. Despite giving both conditions a chance to choose which task to perform, this account would suggest that participants in the deliberative mindset condition nevertheless might have felt less autonomous and less self-motivated than those in the implemental mindset condition.

To assess potential differences in types of motivation between mindset conditions, participants completed Sheldon and Elliot's (1999) four-item measure of self-concordance. Self-concordance is defined as the degree to which people pursue a given goal with feelings of intrinsic interest and identity congruence (Sheldon \& Houser-Marko, 2001) Participants were asked to indicate to what extent they were motivated by four different reasons: external ("somebody else wants you to"), introjected ("you would feel ashamed, guilty, or anxious if you didn't"), identified ("you really believe that it is an important goal to have"), and internal ("for the fun and enjoyment which the goal will provide"). As Sheldon and Elliot (1999) recommend, selfconcordance was calculated by subtracting the sum of the first two items from the sum of the last two items. We also included an item designed to measure perceived autonomy in choice of tasks ("How much autonomy or freedom of choice did you have in making your decision as to which computer task you wanted to do (first)?"). Participants were asked to respond to all questions using 9-item Likerttype scales, with $1=$ not at all and $9=$ very much so. If the findings of this study were a function of differences in motivation rather than mindset, we would expect to find differences in self-concordance and perceive autonomy.

Although there were no significant differences in perceived autonomy, $t(44)=1.41, p=.17, r=.21$, there was a near significant difference between the mindsets in self-concordance, $t(44)=1.737, p=.09, r=.25$. Participants in the implemental mindset $(M=1.09, S D=6.68)$ reported greater goal self-concordance than those in the deliberative mindset $(M=-1.92, S D=5.00)$. Correlational analyses revealed, however, that neither self-concordance nor perceived autonomy were correlated with response latency on the recognition memory task. As a more stringent test, we re-analyzed response latencies by condition with self-concordance and perceived autonomy separately as covariates. A significant effect of mindset on recognition response latencies remained even after adjusting for self-concordance, $F(43)=4.53, p=.04, r=.31$, and for perceived autonomy, $F(43)=4.73, p=.04, r=.31$. These results indicate that although there was a near-significant difference in self-concordance, this difference does not account for the significant difference in response latency on the recognition memory test. Moreover, there were no differences in perceived autonomy, and adjusting for reported levels of perceived autonomy did not change the magnitude of the differences due to mindset on the recognition memory test. This suggests that there is a lack of compelling evidence for a re-interpretation of the results that emphasize differences in the type of motivation participants experienced.

\section{Discussion}

The results of Study 1 provided support for the hypothesis that deliberative and implemental mindsets differ in selective processing of incidental information. Participants in a deliberative mindset evidenced better recognition for incidental words presented during the concentration test than those in an implemental mindset. Although the two conditions did not differ significantly in recognition accuracy, participants in the deliberative mindset condition took less time to recognize those incidental words than did those in the implemental mindset. Shorter recognition response latencies indicate less effort or difficulty in accessing memory traces of information that individuals were previously exposed to. That those in a deliberative mindset required less time to recognize the incidental words suggests that they had engaged in less selective processing than those in an implemental mindset. To rule out alternate explanations for our findings, we included several measures of degree of motivation, self-efficacy, and type of motivation. Analyses of the results from these measures did not provide any support for these re-interpretations. Together, these results provide preliminary evidence that selective processing of incidental information is a distinguishing feature between deliberative and implemental mindsets. Moreover, the effect of mindsets on selective processing appeared to occur pre-consciously (without conscious intent and initiation); that is, even when conscious strategies of information search and exposure are circumvented.

That participants in a deliberative mindset outperformed those in an implemental mindset on the recognition memory task is particularly surprising in light of the instructions that were provided to participants during the mindset manipulation. Recall that participants in a deliber- 
ative mindset were instructed to continue deliberating over their choice of tasks while engaged in the performance task (the computerized d2 Mental Concentration Test). That is, participants were instructed to ask themselves throughout the task "whether the task was right" for them or not. Performing the task while deliberating over a decision can be viewed as a dual-task setting, one that induces cognitive load. This increased load should have made attending to and encoding incidental stimuli much more difficult. Thus, that participants in the deliberative mindset performed better on the recognition memory task than those in the implemental mindset despite increased cognitive load speaks to the power of the pre-conscious cognitive processes associated with each respective mindset.

It should also be noted that the faster response latencies by those in the deliberative mindset was specific to the recognition task and did not generalize to the concentration performance task. That is, although there were significant differences between the two mindsets on the recognition memory test, there were no differences on the $\mathrm{d} 2$ concentration task. This suggests that the faster response latencies by those in the deliberative mindset as compared to the implemental mindset are not indicative of a more general speedup effect, but rather one that is specific to recognition memory of incidental information.

In Study 1, we did not separate the semantic content of the goal that participants were pursuing from the measurement task. The goal that we activated was directly related to the computerized performance task. To replicate Study 1 and to provide more compelling evidence of mindset differences in selective processing, in Study 2, we separated the priming manipulation from the computer task, making sure that the goal we activated was unrelated to performing our measurement task. The transfer of cognitive procedures from one task to an unrelated task is the hallmark of mindset priming (Gollwitzer, 1990). Without demonstrating such a transfer, any differences between conditions are attributable to the differences in the task at hand, rather than the hypothesized cognitive procedures associated with each mindset. To induce mindsets, we used a classic manipulation of deliberative and implemental mindsets used in a number of previous mindset experiments (e.g., Gollwitzer et al., 1990, Study 2; Gollwitzer \& Kinney, 1989, Study 2; Taylor \& Gollwitzer, 1995). We measured pre-conscious information processing of incidental information in the same manner as in Study 1, using performance on a recognition memory test of incidental stimuli presented in the modified d2 Mental Concentration Task as our dependent variable.

\section{Study 2}

\section{Method}

\section{Participants}

Thirty-eight students at New York University participated in the study in exchange for course credit. They were randomly assigned to the deliberative and implemental mindset conditions, and were distributed evenly across conditions.

\section{Materials and procedures}

Participants were run individually, or in groups of two. They were each seated in front of a computer inside a soundproof cubicle, which prevented them from seeing and hearing any other participant. As in Study 1, participants were informed that they were participating in a study that was ostensibly designed to measure their concentration potential. They were told that they would be performing a computer task designed to measure their capacity to focus and attend to information. Prior to performing the computer task, however, participants were asked to complete a pen-and-paper task. They were told that another psychology research laboratory was interested in surveying the student population, and was including a short questionnaire in the same study session. Unbeknownst to the participants, this questionnaire was actually a standard mindset induction used in previous research (Gollwitzer \& Kinney, 1989, Study 2; Taylor \& Gollwitzer, 1995). To ensure that the experimenter remained blind to condition assignment, each packet included a uniform cover sheet.

Mindset manipulation. Participants in the deliberative mindset condition were asked to indicate an unresolved personal problem for which they had not yet come to any decision. As in other mindset studies (Gollwitzer \& Kinney, 1989, Study 2; Taylor \& Gollwitzer, 1995), deliberative mindset participants were first asked to generate the immediate pros and cons involved in making a decision involving a change with respect to their personal problem. They were then asked to record the long-term pros and cons with making such a decision. After listing both short and longterm consequences for a decision to change, they were then asked to repeat the exercise for making a decision that would maintain the status quo with respect to their personal problem.

Those in the implemental mindset condition were asked to indicate a resolvable problem for which they had made a decision or intention to act, but had not yet initiated any direct action. Participants were asked to list five steps that they could take to resolve the personal problem. For each step, they were then asked to indicate when, where, and how each step was to be performed to successfully bring resolution to the problem.

Performance and recognition memory tasks. After completing the mindset manipulation, participants performed the same modified d2 Mental Concentration Task as in Study 1. As before, when they were finished with the computer task, they answered a series of questionnaires. Participants first completed several items designed to account for potential alternative explanations for the predicted pattern of results. These measures and the rationale for including them were the same as in Study 1, with the addition of the 
Positive and Negative Affective Schedule (PANAS; Watson, Clark, \& Tellegen, 1988) to account for alternate explanations based on mood. After completing all measures, participants were probed for suspicion regarding the experimental manipulations and asked to guess what function they thought the words in the $\mathrm{d} 2$ Mental Concentration Task had. When they were finished with the questionnaires (about $5 \mathrm{~min}$ ), participants were presented with the same computerized recognition memory task as in Study 1. They were then carefully debriefed and dismissed.

\section{Results}

\section{d2 mental concentration task performance}

As in Study 1, performance on the d2 Mental Concentration Task by mindset was measured using number of errors and response latencies. Response latencies were transformed using a base-10 logarithmic transformation to correct for excessive skew. A 2 (condition: implemental vs. deliberative) $\times 2$ (trial: word vs. no word) mixed design ANOVA on response latencies revealed only the main effect of trial, $F(1,35)=15.19, p<.001, r=.55$. Participants responded more slowly to words preceded by the presentation of a word $(M=8246 \mathrm{~ms}, S D=2607)$ than those that were not $(M=7570 \mathrm{~ms}, S D=1791)$. There were no significant differences with the number of errors.

\section{Recognition memory task performance}

Performance on the recognition task was analyzed using accuracy and response latencies as dependent variables. As in Study 1, as false alarm rates were equal to zero on some occasions, the traditionally used discriminability index $d^{\prime}$ could not be calculated to measure accuracy. Instead, the nonparametric discriminability index, $A^{\prime}$, was calculated for each participant on the basis of their correct hits and false alarms in the recognition task to measure accuracy (Pollack \& Norman, 1964). All response latencies less than $300 \mathrm{~ms}$ and greater than $3000 \mathrm{~ms}$ were excluded from the analysis (Goschke \& Kuhl, 1993). This accounted for less than $4 \%$ of the data. Response latencies were then logtransformed to correct for excessive skew. Means of these log-transformed response latencies associated only with correct responses were then calculated for each participant and entered into the analysis (Anderson, 1983; Goschke \& Kuhl, 1993). As predicted, recognition accuracy between the mindset conditions was statistically different, $t(36)=2.58, p=.01, r=.40$. Participants in a deliberative mindset $(M=.79, S D=.077)$ correctly recognized more of the words than those in an implemental mindset $(M=.73$, $S D=.061$ ). Analysis of response latencies, however, did not reveal a difference between deliberative $(M=977$, $S D=186)$ and implemental $(M=952 \mathrm{~ms}, S D=177)$ mindsets, $t(36)=.56, p=.58, r=.09$.

\section{Alternate explanations}

As in Study 1, we included several measures to rule out alternate explanations for our results. Accordingly, we used the same measures of commitment, self-efficacy, and intrinsic motivation as in the previous study. There was a nearly significant difference in commitment to performing well on the concentration task, $t(36)=1.66, p=.11$, $r=.27$. Participants in the deliberative mindset $(M=7.56$, $S D=1.29$ ) were more committed than those in the implemental mindset $(M=6.60, S D=2.11)$. There were no significant differences in self-efficacy nor self-concordance. There was a nearly significant difference in perceived autonomy, $t(36)=1.75, p=.09, r=.28$, with deliberative mindset individuals $(M=2.94, S D=2.65)$ reporting greater autonomy than implemental mindset individuals $(M=1.63, S D=1.86)$. Correlational analyses further revealed that self-concordance $(r=.36, p=.03)$ and perceived autonomy $(r=.29, p=.08)$ were correlated with accuracy. Still, adjusting for all of these variables as separate covariates in analyses did not change the results (i.e., the effect of mindset on recognition memory of incidental words). These findings indicate that alternate explanations based on differences between condition as a function of commitment, self-efficacy, and self-concordance are not compelling.

To examine an additional potential account of the results based on mood, we analyzed responses to the PANAS (Watson et al., 1988). An alternate account of the results based on mood would suggest that the instructions to deliberate over one's current problems might have induced a negative mood, which is associated with systematic processing (Bless, Bohner, Schwarz, \& Strack, 1990). The re-interpretation would propose that more systematic processing might have led to enhanced processing of incidental stimuli. Contrary to this mood-based account, results from the PANAS indicate that there were no significant differences in reported positive or negative mood. Mood was also not correlated with recognition accuracy. In line with prior mindset research testing the effects of mindsets on biased inferences (Taylor \& Gollwitzer, 1995), reinterpretations of the results based on differences in mood are not supported by the data.

\section{Discussion}

The results of Study 2, as in Study 1, supported the hypothesis that deliberative and implemental mindsets differ in selective processing of incidental information. In contrast to the results of Study 1, although there were no differences the time it took participants to recognize incidental words, those in a deliberative mindset more accurately recognized incidental words than those in an implemental mindset. This enhanced recognition accuracy conceptually replicates the results of Study 1 and suggests that incidental information presented during the concentration task was processed to a greater degree in the deliberative mindset as compared to the implemental mindset. Together, these two studies indicate that there are differences in pre-conscious processing of incidental information in deliberative and implemental mindsets. 
As in Study 1, we included several items to account for potential alternate explanations of the results, although such re-interpretations were less compelling to begin with due to the change in the manner by which we manipulated deliberative and implemental mindsets. These results indicated that as in Study 1, alternate explanations based on differences between mindset with respect to commitment, self-efficacy, and intrinsic motivation are not well-supported by the data. Re-interpretations based on differences in mood were also not supported by the data. The differences in selective processing in this study, therefore, appear not to be a function of these variables.

Although Studies 1 and 2 provided support for the hypothesis that deliberative and implemental mindsets differ in selective processing of incidental information, it is not clear whether deliberative mindsets are less selective, implemental mindsets are more selective, or a combination of both. In Study 3, we used the same paradigm as Study 2, but included a mindset-neutral control condition. By comparing the selective processing of the two mindsets against this control condition, we hoped not only to replicate the results of the previous studies, but to also gain better understanding of the direction of the effect.

\section{Study 3}

\section{Method}

\section{Participants}

Fifty-four students at New York University participated in the study in exchange for course credit. Participants were randomly assigned to condition: 16 were in the control condition, 16 in the deliberative mindset condition, and 23 in the implemental mindset condition.

\section{Materials and procedures}

Participants were run in groups of $1-4$, and randomly assigned to condition. Each sat in a cubicle equipped with a computer and desk space. Materials and procedures were identical to Study 2, with the addition of a control condition. Instead of completing a mindset manipulation, those assigned to the control condition were asked to list the first 20 thoughts that appeared in their minds. They were encouraged to write down any thought that occurred to them, and to record them regardless of content.

All participants then performed the same modified $\mathrm{d} 2$ Mental Concentration Task as in Studies 1 and 2. After filling out the questionnaires to assess potential alternate explanations of the results, participants performed a recognition memory test of the items presented during the concentration task. Participants were then carefully debriefed and dismissed.

\section{Results}

\section{d2 mental concentration task performance}

As in both Studies 1 and 2, performance on the d2 Mental Concentration Task by condition was measured using number of errors and response latencies. Response latencies were transformed using a base-10 logarithmic transformation to correct for excessive skew. Both 3 (condition: deliberative vs. implemental vs. control) $\times 2$ (trial: word vs. no word) mixed design ANOVAs on errors and response latency revealed only the main effect of trial: $F(2,51)=5.12$, $p=.03, r=.30$, and $F(2,51)=7.13, p=.01, r=.35$, respectively. Participants made more errors $(M=3.55, S D=3.85)$ and took more time $(M=7613 \mathrm{~ms}, S D=1751)$ on trials preceded by presentations of incidental stimuli than those that were not $(M=2.85, S D=3.21 ; \quad M=7116 \mathrm{~ms}$, $S D=1397$, respectively).

\section{Recognition memory task performance}

Performance on the recognition memory test, as in both previous studies, was measured on accuracy and average response latencies. As false alarm rates were equal to zero on some occasions, the nonparametric discriminability index, $A^{\prime}$, was calculated for each participant on the basis of their correct hits and false alarms in the recognition task to measure accuracy (Pollack \& Norman, 1964). All response latencies less than $300 \mathrm{~ms}$ and greater than $3000 \mathrm{~ms}$ were excluded from the analysis (Goschke \& Kuhl, 1993), which accounted for less than $3 \%$ of the data. Response latencies were logtransformed to correct for excessive skew. Only those response latencies associated with correct responses were then averaged for each participant and entered into the analysis (Anderson, 1983; Goschke \& Kuhl, 1993). A one-way ANOVA by condition revealed statistically significant differences between the conditions on accuracy, $F(52)=3.56$, $p=.04, r=.26$. Follow-up focused comparisons indicated that those in the deliberative mindset $(M=.79, S D=.11)$ were significantly more accurate on the recognition memory test than those in the implemental mindset $(M=67$, $S D=.14), t(51)=2.57, p=.01, r=.34$, and control conditions $(M=.67, S D=.17), t(51)=2.03, p=.05, r=.27$. The implemental mindset and control conditions did not differ significantly from each other, $t(51)=.38, p=.71, r=.05$. Analysis of response latencies did not reveal any statistically significant differences between conditions, $F(51)=1.41, p=.25$, $r=.16$. The pattern of means, however, was similar to those of accuracy: those in the deliberative mindset condition were faster $(M=856 \mathrm{~ms}, S D=111)$ than those in the implemental $(M=957 \mathrm{~ms}, S D=199)$ and control conditions $(M=907 \mathrm{~ms}$, $S D=208)$.

\section{Alternate explanations}

As in Studies 1 and 2, we included several measures to rule out alternate explanations for our results. Accordingly, we used the same measures of commitment, self-efficacy, intrinsic motivation, and mood. There were no significant differences in any of the measures by condition, nor were there any significant correlations between these variables and recognition accuracy. Moreover, adjusting for these as covariates did not alter the results reported above. These variables therefore do not appear to tenable bases for alternate interpretations of the data. 


\section{Discussion}

As in Study 2, participants in a deliberative mindset evidenced greater recognition accuracy of incidental words than those in an implemental mindset. This provides further support for the hypothesis that these two mindsets differ in selective processing of incidental information. Moreover, such differences appear to have occurred in the absence of conscious information processing strategies. These results replicate the previous two studies. Results further suggest that the effects of mindsets on selective processing appear to be driven by an enhanced openness and readiness to process incidental information by deliberative mindsets. Those in a deliberative mindset demonstrated greater recognition memory of incidental stimuli presented during the concentration task than both those in the implemental and control conditions. That the implemental and control conditions did not differ suggests that the effect is not driven by enhanced selective processing. Rather, it appears that deliberative mindsets are characterized by preconscious processes that facilitate processing of incidental information available in the environment.

\section{General discussion}

\section{Deliberative mindsets and pre-conscious open-mindedness}

Mindset theory suggests that that the deliberative mindsets should be more open-minded in information processing, whereas implemental mindsets should be more closedminded. As such, we proposed that deliberative mindsets would evidence less selective processing of incidental information, as it behooves individuals to consider all available information when trying to make a good goal decision. In contrast, we hypothesized that the implemental mindset should demonstrate comparatively greater selective processing, as processing of information not directly task-relevant could be distracting and detract from one's ability to focus on performing the task at hand. In three studies, we found evidence for this hypothesis.

In Study 1, participants in a deliberative mindset took less time than those in an implemental mindset to recognize whether or not they had previously been exposed to incidental words presented in a concentration task. In Studies 2 and 3, participants in a deliberative mindset had higher recognition accuracy of these words as compared to those in an implemental mindset. The results from all three studies indicate that deliberative individuals more easily accessed memory traces of information incidental to the ongoing task than implemental individuals. This occurred even when the mindsets induced were unrelated to the performance task that measured the cognitive differences (Studies 2 and 3). This "carry over" effect of mindsets is evidence that strongly suggests that whereas the implemental mindset is more selective, the deliberative mindset is more openminded to incidental information available in one's immediate environment.
Study 3 allowed us to clarify whether the effect of mindset on selective information processing was due to enhanced open-mindedness in the deliberative mindset, enhanced closed-mindedness in the implemental mindset, or both. Results from this study suggested that the change in selective processing as a function of mindset is attributable to less selective filtering of incidental information in the deliberative mindset, as opposed to greater selective filtering in the implemental mindset. Whereas the recognition memory for incidental stimuli of individuals in an implemental mindset mirrored those in a mindset-neutral control condition, recognition was significantly higher among those in a deliberative mindset. That is, the difference in information processing between deliberative and implemental mindsets is driven by a greater receptivity to incidental information by the deliberative mindset. As hypothesized by mindset theory, the deliberative mindset, to facilitate choosing between goals, engages cognitive procedures that open the mind to task-incidental stimuli, thus considering carefully all available information that might be relevant to a goal decision.

Remarkably, the results of these studies suggest that changes in selective processing as a function of mindset occur pre-consciously. By pre-conscious, we refer to cognitive processes that initiated and operate outside of conscious intent (Bargh, 1994). Researchers have argued that reactions to stimuli that require a response within $300 \mathrm{~ms}$ are not consciously controlled (e.g., Bargh \& Chartrand, 2000; Greenwald \& Banaji, 1995; Neely, 1977). Participants in all studies reported here were presented with incidental stimuli for only $300 \mathrm{~ms}$ prior to the primary performance task materials. Moreover, the presentation of these stimuli was random and located in the center of the participants' visual fields. Together, the short exposure times and random central presentation of the incidental stimuli should have precluded conscious strategies of avoidance, thus any increment in processing should be considered outside of conscious intent. These data suggest that the act of deliberating and making a goal decision produces dramatic changes in the cognitive processing of information even when individuals do not intend such changes. In our view, these pre-conscious changes occur to support individuals' progression through the various stages of goal pursuit.

\section{Speed and accuracy}

The significant differences between the mindsets on recognition memory were more evident with response latencies in Study 1, and with accuracy in Studies 2 and 3. Although we conceptually replicated support for our predictions across three studies, the findings were not apparent in the same measure of information processing (i.e., accuracy vs. response latencies). As both measures are well-established indices of information processing (Anderson, 1983), we believe that this inconsistency does not necessarily undermine the support for our hypotheses. To be fair, as is evident in the summary table of results (Table 1), the pattern 
Table 1

Summary table of results for studies 1-3

\begin{tabular}{|c|c|c|c|c|c|c|c|c|c|}
\hline & \multicolumn{9}{|c|}{ Mindset condition } \\
\hline & \multicolumn{3}{|c|}{ Deliberative } & \multicolumn{3}{|c|}{ Implemental } & \multicolumn{3}{|c|}{ Control } \\
\hline & $M$ & $S D$ & $N$ & $M$ & $S D$ & $N$ & $M$ & $S D$ & $N$ \\
\hline \multicolumn{10}{|c|}{ Accuracy $\left(A^{\prime}\right)$} \\
\hline Study 1 & .77 & .078 & 24 & .75 & .060 & 22 & - & - & \\
\hline Study 2 & .79 & .077 & 14 & $.73_{\mathrm{b}}$ & .061 & 14 & - & - & \\
\hline Study 3 & $.79_{\mathrm{ab}}$ & .11 & 16 & $.67_{\mathrm{b}}$ & .14 & 23 & $.67 \mathrm{a}$ & .17 & 16 \\
\hline \multicolumn{10}{|c|}{ Response latency (ms) } \\
\hline Study I & $974 \mathrm{a}$ & 160 & 24 & 1162 a & 380 & 22 & - & - & \\
\hline Study 2 & 977 & 186 & 14 & 952 & 177 & 14 & - & - & \\
\hline Study 3 & 856 & 111 & 16 & 957 & 199 & 23 & 907 & 208 & 16 \\
\hline
\end{tabular}

Note. Subscript letters note statistically significant differences (a: $p=.05$, b: $p=.01$ )

of results for accuracy in Study 1, although not statistically significant, was consistent with those in Studies 2 and 3. Moreover, the differences in response latency in Study 3, also not statistically significant, were consistent with the findings from Study 1. To test whether the effect of deliberative vs. implemental mindsets differed between studies, and whether the aggregated findings were statistically significant across the three studies on both measures, we performed a series of meta-analyses as recommended by Rosenthal and Rosnow (1991). These analyses revealed that the effects of mindsets in of the three studies did not differ significantly from each other neither with respect to accuracy, $\chi^{2}(2)=1.45, p=.49$, nor response latency, $\chi^{2}(2)=3.81, p=.15 .^{5}$ Moreover, aggregating the findings across all three studies, deliberative mindsets led to greater accuracy, $Z=3.49, p<.01$, and faster response latencies, $Z=1.89, p=.06$, than implemental mindsets. Thus, from a meta-analytic perspective, the results from these studies may not be as discrepant as appears at first glance and reveal mindset differences with both accuracy and speed measures.

Nevertheless, we are cognizant that a conservative interpretation of these three studies suggests a need to reconcile why at times the differences in mindsets are evidenced in response latencies, whereas at other times they are revealed with accuracy. This apparent discrepancy may reflect differences in the sensitivity of speed and accuracy as performance measures resulting from differences in how the mindsets were induced between the two studies. Research has shown that even subtle changes in the framing of a task can lead to change the sensitivity of speed and accuracy measures, even when participants are explicitly instructed to be concerned with both speed and accuracy. Förster, Higgins, and Bianco (2003), for example, have shown that both chronic and situational regulatory foci (prevention vs. promotion orientations) can change whether individuals display performance differ-

\footnotetext{
5 In these analyses, to account for differences in the $\mathrm{N}$ in each study, the results of each study was weighted by the $N$ (Rosenthal \& Rosnow, 1991). Unweighted analyses revealed similar results.
}

ences with respect to speed or accuracy. They found that despite giving participants identical instructions (emphasizing both speed and accuracy as equally important measures), performance differences were more evident with speed as a performance measure when participants were in a promotion focus (a motivational orientation toward ideals and gains/nongains), and more evident with accuracy as a performance measure when participants were in a prevention focus (a motivational orientation toward oughts and nonlosses/losses). The relative sensitivity of speed and accuracy measures in detecting performances differences, therefore, can be a function of the strategic motivational orientations of individuals.

It seems plausible to suggest that research participants in Study 1 may have been in a more promotion focus, whereas those in Studies 2 and 3 may have been in more prevention focus. In Study 1, participants were asked to choose which of two tasks they wanted to perform. The ability to decide which task one "wanted" to perform may have been a subtle manipulation of a promotion focus, which stresses one's wants, wishes, and ideals (Higgins, 1997). On the other hand, in Studies 2 and 3, participants were not given a choice of tasks. As a result, they may have felt more "required" to perform the concentration task, which may have subtly induced a prevention focus, which stresses one's obligations, responsibilities, and oughts (Higgins, 1997). Empirically supporting these suggestions, participants in Study $2(M=-4.68, S D=5.32)$ and Study $3(M=-4.44, S D=5.25)$ reported much less self-concordance than those in Study $1(M=-.48$, $S D=6.0$ ). The more negative one's self-concordance score is, the more one is motivated by extrinsic ("somebody else wants me to") and introjected motivations ("I would feel ashamed, guilty, or anxious if I didn't"). Shame, guilt, and anxiety are motivational responses associated with concerns about one's responsibilities and duties, and reflect greater prevention regulatory focus (Higgins, 1997). External sources of motivation are also associated with greater prevention regulatory focus, particularly when linked to shame and guilt (the "ought-other" self; Higgins, 1996). In sum, these data support interpreting the shift in sensitivity of response latencies and accuracy as performance measures in detecting differences between mindsets from Study 1 to Studies 2 and 3 as a function of strategic orientations induced by the use of different mindset inductions. That is, response latencies may have been more sensitive to performance differences in Study 1 due to a subtle promotion focus framing, whereas accuracy may have been more sensitive to performance differences in Studies 2 and 3 due to a subtle prevention focus framing.

\section{Encoding vs. retrieval}

Although the three studies reported in this paper indicate that deliberative and implemental mindsets differ in selective processing of goal-incidental information, it is not clear whether such selective processing occurs at 
encoding or retrieval. That is, selective information processing differences between the mindsets may be due to different attentional processes at initial exposure to incidental information, or by differences in the ease of accessing information from memory at the time of recognition. Both selective encoding and selective retrieval of incidental information are consistent with mindset theory's proposition that deliberative mindsets are more open-minded than implemental mindsets. Future research that addresses whether selective processing occurs at encoding, retrieval, or both, however, is clearly warranted.

\section{Implications for motivation and cognition research}

\section{Mindsets}

By providing evidence for differences in pre-conscious cognitive processing of incidental information, these three studies extend and build upon previous mindset research. In particular, these results develop previous work by Heckhausen and Gollwitzer (1987, Study 2) in demonstrating that deliberative mindsets are associated with greater open-mindedness than the implemental mindset. As stated in the Introduction, this previous experiment focused on testing differences in working memory associated with each mindset, and found that the deliberative mindset led to broader working memory spans than the implemental mindset. The present three studies suggest that in addition to enhancing working memory, the deliberative mindset leads to greater selective processing of information that is incidental to one's goals. Moreover, the present data further suggest that such reduced selectivity occurs pre-consciously; that is, without conscious initiation or intent.

Thus, in addition to the mindset features of cognitive tuning and biased inferences, these results suggest that a third distinguishing feature of mindsets is how information available in one's environment is processed. Deliberative mindsets, as compared to implemental mindsets, are characterized by pre-conscious cognitive procedures that lead to decreased selective filtering and thus to greater openness. Together, these results suggest that there are a number of cognitive procedures that support and promote successful goal attainment. These cognitive procedures are activated at appropriate stages of goal pursuit to help individuals effectively and efficiently accomplish the tasks necessary at each phase (Gollwitzer, 1990; Gollwitzer \& Bayer, 1999).

Throughout this paper, we have used the terms "incidental" and "central" to refer to stimuli that differ in their instrumentality in achieving an ongoing task. It is possible, however, that mindsets lead to differences in processing of information that is incidental vs. central in another meaning. Mindsets may lead to changes in one's breadth of attention, thus increasing or decreasing the processing of stimuli presented at the periphery of the visual field. The general open-mindedness of the deliberative mindset may not only lead individuals to process task-incidental information to a greater extent than implemental mindsets, but also to information that is located in peripheral areas of the visual field. The studies presented here do not address this question, as all information was presented in the center of the visual field. Thus, the possibility that the deliberative mindset expands one's attention to peripheral visual areas in addition to one's consideration of information incidental to an ongoing task remains to be tested.

\section{Goal-relevant information processing}

The three studies reported here also complement other findings reported by researchers interested in the intersection of motivation and cognition. For example, these studies dove-tail with recent research by Moskowitz (2002), who has provided evidence for greater attention to goal-relevant information among individuals who had goals made cognitively accessible as compared to those who did not. Not only did individuals attend to goal-relevant stimuli to a greater degree when they were primed with goals, but these effects also appeared to be pre-conscious or outside of conscious intent. The notion that implemental individuals (those involved in goal implementation as a result of heightened goal accessibility) pay greater attention to goal-relevant information is consistent with our proposal that selective information processing differs whether one is deliberating between goals or actively implementing goals. It remains to be demonstrated empirically, however, whether mindsets produce changes in the automatic processing of goal-relevant stimuli, as the studies reported here addressed only the processing of stimuli incidental to an ongoing task. Nevertheless, Moskowitz's findings suggest that not only might deliberative mindsets make individuals more receptive to available information that may potentially inform their goal decisions, implemental mindsets may also make individuals more sensitive to stimuli directly relevant to the chosen goal.

\section{Goal shielding}

It is important to distinguish the selective information processing studied in this paper from extant work on "goal shielding," the protection of a goal intention from competing intentions (Kuhl, 1984; Shah, Friedman, \& Kruglanski, 2002). By preventing individuals from falling prey to the temptation of alternative goal pursuits, goal shielding is theorized to be a crucial aspect of successful goal attainment. Goal shielding differs from the selective information processing examined in this paper as it refers specifically to the inhibition of competing goal intentions. The selective processing reported here refers to the processing of information that is incidental, but not necessarily antagonistic, to one's current task goal. This information potentially could be useful, but its connection is not readily apparent. In our view, goal shielding and the selective information processing we have studied here are manifestations of the same, more general open- 
and closed-minded orientations of deliberative and implemental individuals. Thus, we would predict that implemental mindsets to be characterized more by goal shielding than deliberative mindsets. Prior to committing to a goal, goal shielding is nonsensical as there is no goal intention to protect. Instead, one should be open to all goal possibilities and carefully deliberate over which to pursue. Once a goal is chosen and goal pursuit efforts engaged, however, one should selectively inhibit competing goal possibilities, as they may derail attainment of the chosen goal. Research on goal shielding has yet to address this possibility, as it has not incorporated differences in deliberative and implemental phases of goal pursuit (e.g., Shah et al., 2002).

\section{Conclusion}

The recent convergence of motivation and cognition in social psychology has emphasized the role of cognitive processes in goal pursuit. In this paper, we have presented three studies that corroborate this claim. Deliberative and implemental mindsets differ in selective information processing, with the deliberative mindset more open and receptive to all available information. The continued study of cognitive procedures, like selective information processing, which support goal pursuit (even without conscious awareness) promises to illuminate the remarkable ability of individuals to select appropriate goals, and to implement these decisions through planning and action.

\section{References}

Anderson, J. R. (1983). The architecture of cognition. Cambridge, MA: Harvard University Press.

Armor, D. A., \& Taylor, S. E. (2003). The effects of mindset on behavior: Self-regulation in deliberative and implemental frames of mind. Personality and Social Psychology Bulletin, 29, 86-95.

Bargh, J. A. (1994). The Four Horsemen of automaticity: Awareness, efficiency, intention, and control in social cognition. In R. S. Wyer Jr. \& T. K. Srull (Eds.), Handbook of social cognition (2nd ed., pp. 1-40). Hillsdale, NJ: Erlbaum.

Bargh, J. A., \& Chartrand, T. (2000). The mind in the middle: A practical guide to priming and automaticity research. In H. T. Reis \& C. M. Judd (Eds.), Handbook of research methods in social and personality psychology (pp. 253-285). New York: Cambridge University Press.

Bless, H., Bohner, G., Schwarz, N., \& Strack, F. (1990). Mood and persuasion: A cognitive response analysis. Personality and Social Psychology Bulletin, 16, 331-345.

Brickenkamp, R. (1981). Test d2 (7th ed.). Goettingen, Germany: Hogrefe.

Förster, J., Higgins, E. T., \& Bianco, A. T. (2003). Speed/accuracy decisions in task performance: Built-in trade-off or separate strategic concerns? Organizational Behavior and Human Decision Processes, $90,148-164$.

Gagné, F. M., \& Lydon, J. E. (2001). Mind-set and close relationships: When bias leads to (in)accurate predictions. Journal of Personality and Social Psychology, 81, 85-96.

Gollwitzer, P. M. (1990). Action phases and mind-sets. In E. T. Higgins \& R. M. Sorrentino (Eds.), Handbook of motivation and cognition: Foundations of social behavior (Vol. 2, pp. 53-92). New York: Guilford Press.

Gollwitzer, P. M., \& Bargh, J. A. (1994). The psychology of action: Linking motivation and cognition to behavior. New York: Guilford.
Gollwitzer, P. M., \& Bayer, U. (1999). Deliberative versus implemental mindsets in the control of action. In S. Chaiken \& Y. Trope (Eds.), Dual process theories in social psychology (pp. 403-422). New York: Guilford.

Gollwitzer, P. M., Fujita, K., \& Oettingen, G. (2004). Planning and the implementation of goals. In R. Baumeister \& K. Vohs (Eds.), Handbook of self-regulation research (pp. 211-228). New York: Guilford.

Gollwitzer, P. M., \& Kinney, R. F. (1989). Effects of deliberative and implemental mind-sets on illusion of control. Journal of Personality and Social Psychology, 56, 531-542.

Gollwitzer, P. M., Heckhausen, H., \& Steller, B. (1990). Deliberative and implemental mind-sets: Cognitive tuning toward congruous thoughts and information. Journal of Personality and Social Psychology, 59, $1119-1127$.

Goschke, T., \& Kuhl, J. (1993). Representation of intentions: Persisting activation in memory. Journal of Experimental Psychology: Learning, Memory, and Cognition, 19, 211-1226.

Greenwald, A. G., \& Banaji, M. R. (1995). Implicit social cognition: Attitudes, self-esteem, and stereotypes. Psychological Review, 102, 4-27.

Heckhausen, H., \& Gollwitzer, P. M. (1987). Thought contents and cognitive functioning in motivational versus volitional states of mind. Motivation and Emotion, $11,101-120$.

Higgins, E. T. (1996). The "self digest": Self-knowledge serving self-regulatory functions. Journal of Personality and Social Psychology, 71, 1062 1083.

Higgins, E. T. (1997). Beyond pleasure and pain. American Psychologist, $52,1280-1300$.

Kucera, H., \& Francis, W. N. (1967). Computational analysis of present-day American English. Providence, RI: Brown University Press.

Kuhl, J. (1984). Volitional aspects of achievement motivation and learned helplessness: Toward a comprehensive theory of action control. In B. A. Maher (Ed), Progress in experimental personality research (Vol. 13, pp. 99-171). New York: Academic Press.

Kruglanski, A. W., Shah, J. Y., Fishbach, A., Friedman, R., Chun, W. Y., \& Sleeth-Keppler, D. (2002). A theory of goal systems. In M. P. Zanna (Ed.), Advances in experimental social psychology (Vol. 34, pp. 331378). San Diego, CA: Academic Press.

Moskowitz, G. B. (2002). Preconscious effects of temporary goals on attention. Journal of Experimental Social Psychology, 38, 397-404.

Neely, J. H. (1977). Semantic priming and retrieval from lexical memory: Roles of inhibitionless spreading activation and limited-capacity attention. Journal of Experimental Psychology: General, 106, 226-254.

Oettingen, G., \& Gollwitzer, P. M. (2001). Goal setting and goal striving. In A. Tesser \& N. Schwarz (Eds.), Blackwell handbook in social psychology: Intraindividual processes (Vol. 1, pp. 329-347). Oxford, England: Blackwell.

Pollack, I., \& Norman, D. A. (1964). A nonparametric analysis of recognition experiments. Psychonomic Science, 1, 125-126.

Puca, R. M., \& Schmalt, H.-D. (2001). The influence of the achievement motive on spontaneous thoughts in pre- and post-decisional action phases. Personality and Social Psychology Bulletin, 27, 302-308.

Puca, R. M. (2001). Preferred difficulty and subject probability in different action phases. Motivation and Emotion, 25,307-326.

Rosenthal, R., \& Rosnow, R. L. (1991). Essentials of behavioral research: Methods and data analyses (2nd ed.). New York: McGraw Hill.

Shah, J. Y., Friedman, R., \& Kruglanski, A. W. (2002). Forgetting all else: On the antecedents and consequences of goal shielding. Journal of Personality and Social Psychology, 83, 1261-1280.

Sheldon, K. M., \& Elliot, A. J. (1999). Goal striving: Need satisfaction, and longitudinal well-being: The self-concordance model. Journal of Personality and Social Psychology, 76, 482-497.

Sheldon, K. M., \& Houser-Marko, L. (2001). Self-concordance, goal attainment, and the pursuit of happiness: Can there be an upward spiral? Journal of Personality and Social Psychology, 80, 152-165. 
Snodgrass, J. G., Volvovitz, R., \& Walfish, E. R. (1972). Recognition memory for words, pictures, and words + pictures. Psychonomic Science, 27, 345-347.

Sorrentino, R. M., \& Higgins, E. T. (1986). Handbook of motivation and cognition (Vol. 1): Foundations of social behavior. New York: Guilford.
Taylor, S. E., \& Gollwitzer, P. M. (1995). The effects of mindsets on positive illusions. Journal of Personality and Social Psychology, 69, 213-226.

Watson, D., Clark, L. A., \& Tellegen, A. (1988). Development and validation of brief measures of positive and negative affect: The PANAS scales. Journal of Personality and Social Psychology, 54, 1063-1070. 